\title{
Alignment between the Learning Objectives and Examinations: A Comparative Analysis of High-School Physics Curricula in Myanmar and South Australia
}

\author{
Robert Matthews ${ }^{1 *}$, Wai Wai Kyi ${ }^{2}$ \\ ${ }^{1}$ Senior Lecturer School of Education, University of Adelaide, Adelaide, Australia \\ ${ }^{2}$ Lecturer Sagaing University of Education, Sagaing, Myanmar
}

DOI: $10.36348 /$ sb.2019.v05i11.011 $\quad$ | Received: 21.11.2019 | Accepted: 28.11 .2019 | Published: 30.11 .2019

*Corresponding author: Robert Matthews

\section{Abstract}

The purpose of this study is to analyse and compare the alignments of the Myanmar and the South Australian high-school physics curricula in relation to their respective learning objectives, learning outcomes and examinations. In both instances curricula from the completing year of high-school are examined: the Grade 10 Physics curriculum in Myanmar, and the Year 12 Physics curriculum in South Australia. 2018 materials, those most recently available, have been used for both Myanmar and South Australia. A slightly modified Porter's alignment model is used to analyse curriculum alignment with objectives and assessment. The Myanmar physics curriculum demonstrated an alignment index with outcomes and examination of 0.58 and in the case of South Australia, the alignment index was 0.53. Both alignment indices were significantly lower than the critical values (0.839) and (0.884) at the 0.05 level. Most objectives and questions over-represented lower level cognitive skills. In Objectives: Remember (35\%) and Understand (51\%) for Myanmar, and Understand (54\%) and Apply (31\%) for South Australia; in Examinations, Remember (49\%) and Apply (33\%) in Myanmar and Understand (24\%) and Apply (49\%) in South Australia. Concerns are raised over diminished expectations of, and for, student success and discussion given on issues of validity in learning outcomes and assessments. Recommendations are then made to adjust and improve the alignment of materials used the two contexts of physics instruction.

Keywords: Alignment, curriculum, assessment, objectives, Porter's Alignment model.

Copyright @ 2019: This is an open-access article distributed under the terms of the Creative Commons Attribution license which permits unrestricted use, distribution, and reproduction in any medium for non-commercial use (NonCommercial, or CC-BY-NC) provided the original author and source are credited.

\section{INTRODUCTION}

International comparison in attainment outcomes (through TIMMS, PISA, etc) have proven an invaluable tool fostering reform in science education since the 1990s [1-3]. Such comparison has promoted greater scrutiny of the curricula and examination systems that belie these attainment outcomes [4]. An important measure to understand the effectiveness of such content delivery and its assessment is the alignment of a curriculum with its objectives and assessment outcomes. Such studies have been carried out in United States [5-7] in Africa [8, 9], in China [1012] and in Singapore [13] and have assisted policy makers and curriculum and assessment developers to enhance their materials coherence and consistency. This paper seeks to explore such alignment in the physics curricula and examination materials in the two previously unresearched contexts of Myanmar and
South Australia in comparison with previously carried out alignment studies (from New York, China and Singapore). Myanmar was selected for its curriculum is soon to undergo major rewriting and consequently to benchmark its current alignment would assist curriculum writers by indicating desirable areas of improvement. In the South Australian context a national curriculum was recently introduced over the top of existing materials with no attempt to test their mutual alignment.

Many researchers have pointed out the advantages of having a strong constructive alignment between the teaching and learning process. Biggs and Tang [14] defined constructive alignment - the interrelationship between what we want (objectives), how we teach (teaching-learning activities), and how we assess (summative assessments), thus resulting in 
much more effective teaching. They argue such alignment assists students to understand learning and assessment expectations, thus supporting learning through appropriate learning activities as all components in the system are in maximum consistency, addressing the same agenda and supporting each other. Others have emphasized that constructive alignment aids the teacher through coherent delivery of instructional practice with the desired content, leading to increased student achievement $[15,7]$. In order to maximize effective learning, a constructive alignment should exist to complement learning outcomes, teaching and learning approaches, and assessment techniques that can facilitate students learning and communicate clear expectations $[16,18]$. Or as suggested by Orlich, Harder, Callahan, Trevisan, and Brown [19]. When all components of the curriculum, objectives, instruction, and assessment, are in alignment, student learning improves significantly as teachers test what they teach and they teach what is in the objectives.

However strong alignment is no guarantee of high student achievement as even high consistency may only support lower order student attainment if the objectives and assessment are of low cognitive complexity. It is thus important to not only analyse the alignment between standards and assessments, but also to examine the cognitive complexity of standards and questions utilised in examinations. As Kitchen and Wilson [20] argue, even if assessment items are well aligned with educational objectives, they can only be regarded as useful tools if they are of high quality.

\section{Context}

In Myanmar, Physics is taught in the scope of "Natural Science" during the first four years of basic education and in the scope of "Applied science" in lower secondary education. In high school, students may elect to study physics as a stand-alone subject. Students in Myanmar can choose one of 3 options when they enter high school: science, arts, or a combination of science and arts. They have to take 3 compulsory subjects: Myanmar, English and Mathematics. However, science specialized students have to take 3 additional subjects: Physics, Chemistry and Biology. Students who combine science and arts can choose 2 science subjects and one arts subject or 1 science subject and two arts subjects. Students have to take an external written examination at the end of the academic year and this is the only examination they have to sit in Grade 10 before University entry. All students have to pass all six subjects (40\% for pass mark for each subject) in their exit examination. Based on their specialized subjects and the scores or results in their matriculation examination, students are directed to the universities they can apply for. High school physics content includes 13 chapters in total and they are categorized into six separate fields: Mechanics; Heat;
Waves and Sound; Optics; Electricity and Magnetism; and Modern Physics.

All students in South Australia learn Physics in their Year 8, 9 and 10 studies under a general science topic. In Years 11 and 12, students may elect to study Physics as a stand-alone subject similar to the Myanmar context. Upon completion of Year 12 students finish their schooling, obtaining their South Australian Certificate of Education (South Australian Certificate of Education [SACE], [22]. Whereas in Myanmar the final school year of physics studies is $100 \%$ exam assessed, in South Australia the external examination counts for $30 \%$ of the year's grade whilst the remaining $70 \%$ arises from school-based assessments such as tests, laboratory work and written assignments. In our study we confine our analysis to the external examination as the school based component is not defined adequately for our purposes. The objectives for South Australia's Year 12 physics curriculum are set at the national level (Australian Curriculum Assessment Reporting Authority [ACARA], [21]. Australia's Physics curriculum has four main units: Thermal, Nuclear and Electrical Physics; Linear Motion and Waves; Gravity and Electromagnetism; and Revolutions in Modern Physics.

\section{Previous Research on Alignment Studies}

There have been a number of studies analysing the coherence between content, instruction, standards, objectives, assessments and tests by using various alignment models. These alignment studies have been conducted across countries ranging from the United States, South Africa, China and Singapore, and across subjects including science, earth science, physical science, physics, biology and mathematics.

These studies determined the significance of alignment indices based on a range of different criteria - see below for references. For example, some studies created an algorithm in Micro visual basic application and the alignment index is decided to be significant against the value derived from a random sampling distribution. Other studies, utilised the mean value and/or critical value derived from the simulation study of Fulmer [6], and these critical values can be applied for different cell values and different standard and test points. Our study also utilised these critical values to determine the alignment indices. There are some studies, however, that did not use any criteria to qualify the strength of the alignment index. They compared the computed alignment indices against an average of value of 0.5 (as an alignment index ranges from 0 to 1 ). The following summary of results from numerous studies shows a generally consistent finding of misalignment and low alignment across years, states, grades and subjects. 
Liu and Fulmer [13] conducted an alignment study of the science curriculum and assessment in selected NY state schools, the so-called Regents exams, of January 2004, January 2006 and June 2006 for both Physics and Chemistry. They found alignment indices: $0.805,0.834,0.787$ for Physics; and 0.597, 0.731, 0.692 for Chemistry. They considered their alignment indices to be high based on the intuition that these are above the medium value of 0.5. But, according to a reexamination by Fulmer [6], it was found that the average alignment index for Physics was equivalent to the mean (0.80) and that for Chemistry was lower than the mean value. Liang and Yuan [11] examined alignment of Chinese national physics curriculum guidelines and $12^{\text {th }}$ grade exit examinations in 2006 and 2007 and found low alignment indices, 0.37 for 2006 and 0.41 for 2007. They argued that even though alignment indices were found to be low, these measures cannot be simply interpreted as good or bad as the Porter alignment index depends on the number of cells. However, when these are compared with the critical value (0.59) for 108 cells and 120 standard points by Fulmer [6], they seemed well below the critical value to be significant at the 0.05 level.

Liu et al. [2] recently conducted an international alignment study, comparing materials from three different countries. They investigated the alignment between the content of physics standards and the standardized tests for Jiangsu (China), New York (United States), and Singapore. This study revealed low alignment indices in Singapore and China and high alignment in New York. Based on a critical value (0.78) from the random sampling distribution, the study found there was a significant alignment index $(\mathrm{p}<0.05)$ for New York of 0.80 and low alignment indices $(p>0.05)$ for China and Singapore of 0.67 . This study also found that Singapore's standardized test had substantial coverage of Bloom's cognitive levels, Analyse, Evaluate and Create levels, whilst the New York test did not measure beyond the Apply level and the Jiangsu did not go beyond the Analyse level. This shows that New York physics materials, despite having high alignment, did so at a low cognitive level, whilst Singapore materials demonstrated testing at a high cognitive level despite low alignment. Edwards [8] also conducted an alignment study between the Grade 12 Physical sciences examination in 2008 and 2009 and South Africa's core curriculum and found average alignment indices of 0.783 for Physics and 0.628 for Chemistry. The alignment index for Physics was determined to be significant at the 0.05 level based on the critical value (0.78) shown by Liu et al. [2], whilst that for Chemistry was lower than the critical value and not significant. Polikoff et al. [7] also examined the degree of alignment between state assessment of student achievement and state content standards. They found that the overall average alignment index between tests and standards was 0.19 for ELAR (English,
Language, Arts and/or Reading), 0.27 for Mathematics and 0.26 for Science, indicating low alignment with indices across grades and states ranging from 0.01 to 0.47 . They commented that no alignment index was greater than 0.5 for any grade, subject or state. Ndlovu and $\mathrm{Mji}$ [9] calculated the alignment between the South Africa Revised National Curriculum Statements (RNCS) and the Grade 8 TIMSS assessment frameworks for Mathematics. They identified a low alignment index of 0.751 , which was significantly lower than the critical value of alignment index (0.93) established by Fulmer [6].

$\mathrm{Lu}$ and Liu [12] explored the alignments between China's national High School Biology curriculum standards and the 2009 standards-based High School Exit Exam in the four provinces: Gaungdong, Shandong, Hainan and Ningxia. Their study found that the 2009 standardized tests conducted in all four provinces were not aligned with the national Biology curriculum standards. The alignment indices were 0.5657 for Guangdong, 0.6081 for Shandong, 0.4571 for Hainan and 0.5605 for Ningxia. These values were found to be lower than the critical values derived from random sampling distribution: 0.640 for 120 test points and 0.653 for 120 test points and so not significant. Contino [5] conducted an alignment study between curriculum and assessment in the New York State Earth Science Standards-Based system and found a slightly low alignment index of 0.35 due to different emphases on cognitive levels. To determine the significance of the alignment, an algorithm was created and based on random sampling distribution; the critical value of an alignment index (0.78) is needed in order for it to be statistically significant. So, it is apparent that the computed index of 0.35 is well below the critical value. Liang et al. [10] examined the consistency between China's national science standards and their standardized exams in secondary schools and further analysed alignment looking for regional variation across Guangdong, Jiangsu, Shandong, Hainan, and Ningxia provinces. The results found values of alignment indices for Senior High schools in Jingsu province range from 0.42 to 0.47 in 2007-2011. None of the indices are statistically significant at the 0.05 level as the critical value for the $8 * 6$ matrix is 0.67 [6]. In addition, alignment index at Junior high school level range from 0.52 to 0.60 and these are also well below the critical values 0.78 at the 0.05 level for $5^{*} 6$ matrix [6].

\section{MATERIAL AND METHODS}

This study aims to extend these previous alignment studies by exploring the alignment of materials for the final year of schooling in physics for two new contexts: Myanmar and Australia. The physics materials used are the centralized curriculum and assessment of Myanmar and the National Curriculum of Australia and the assessment system in South Australia 
(Australia provides standards at the national level, but assesses only at the state level). This study analysed the alignment between Myanmar and South Australia's learning objectives, learning outcomes, and examinations, and the quality of the statements of objectives and questions. The Myanmar assessment items were sourced privately, the learning objectives were obtained from the published teachers manuals. The South Australian assessment items are available online [21] as are the learning objectives which are taken from the Australian National Curriculum documents (Australian Curriculum Assessment Reporting Authority [22].

\section{Framework of Analysis: Porter's Alignment Model}

The framework to analyse the alignment between objectives and assessment is the slightly modified Porter's alignment model that replaces Porter's five categories of cognitive demand with the six categories of the revised Bloom's taxonomy, i.e., remember, understand, apply, analyse, evaluate and create [23]. Porter's model focuses on the analysis of alignment in both the cognitive- and content-based domains, derived on data from the Surveys of Enacted Curriculum (SEC) [24]. Originally, there were three dimensions in Porter's taxonomy: content dimension, cognitive dimension and mode of presentation. However, the third dimension, mode of presentation, has since been excluded at it was found to be of little use in the analysis of alignment, thus leaving only two dimensions (cognitive dimensions and content dimension) in the final version of the Porter taxonomy table [25]. This table is a combination of Bloom's taxonomy and Porter's taxonomy that can categorize both the content and complexity of terms in the cognitive domain and has been used successfully in previous studies to analyse the alignment between standards, instruction and assessments. Porter's alignment model can be applied to analyse the degree of match between any two curriculum, standards, instructions and assessment items [2].

To determine alignment between curriculum standards and a specific test, firstly, two tables are created, one representing the objectives and the other the test. Each of these tables uses a two-dimensional matrix, in which the rows represent topics and themes in the standards, and the columns represent levels of cognitive demand in the tests (such as Table 1 for the Myanmar Physics Objectives and Table 2 for the Assessment). The values in each cell are standardized as fractional proportions of the grand total of all cell values. Thus indicating the proportion of total content in the standards document (or exam items) that emphasises that particular combination of topic and cognitive demand. This is then followed by a calculation of the absolute discrepancy value between the corresponding cells in each table. Finally, the
Porter's alignment index, $\mathrm{P}$, is calculated using the following equation [25]:

$$
\mathrm{P}=1-\frac{\sum_{i=1}^{n}|(X i-Y i)|}{2}
$$

Here, the value of $\mathrm{P}$ ranges from 0 (indicating no alignment) to 1 (indicating perfect alignment), $\mathrm{X}$ denotes cell proportions in Table $X$ (e.g., the standardized exam matrix), Y denotes cell proportions in Table Y (e.g., the curriculum standards matrix), "n" represents the total number of cells, and $i$ refer to a specific cell in each matrix. After calculating the alignment indices, the results for statistical significance are further tested through the examination of estimated critical values derived from Fulmer's [6] simulation study.

\section{Coding and Data Analysis}

In this study, a coding scheme was employed following Porter's alignment model with utilisation of the revised Bloom's taxonomy. Two coders: the researcher and a professor with more than 15 years of experience in physics teaching coded each unit. The coding was independently completed using verbs in six levels of taxonomy based on the revised Bloom's taxonomy [26]. After coding independently, the correlation coefficient between the cell values of the two tables was calculated to see the inter-rater reliability using Cohen's Kappa index of reliability. The inter-rater reliability (IRR) was $0.90(n=30, p<.000)$ for Myanmar objectives, $0.95(\mathrm{n}=30, \mathrm{p}<.000)$ for Myanmar examination, $0.85(n=30, p<.000)$ for Australian outcome statements, and $0.79(n=30, p<.000)$ for South Australian written examination. The reliability values showed good inter-rater reliabilities. Subsequently, the two coders, coded jointly to develop the final values of each document for analysis by negotiating agreement for any earlier coding differences. The unit of analysis, in this case, the noun and verb phrases in the statements of objectives and questions, was identified prior to analysis of documents. Finally, the content was quantified, which involved a calculation of the frequency of content in each statement and question.

\section{Two Factors Affecting Strength of Alignment}

There are two factors affecting the strength of alignment between items. The first is the number of cells in tables showing the standards and assessment. Tables having a greater number of cells are more likely to yield lower values than those having fewer cells as an increase in the number of a table's cells tends to cause a discrepancy between the ratios, resulting in lower index values [6]. The second factor contributing to the strength of the index values is the quantity of standards statements, or of the assessment items being coded [6]. If there are more statements or items in the alignment studies, the alignment index tends to be larger for studies having the same number of cells. So, it is important to use standard critical values for comparing 
Robert Matthews \& Wai Wai Kyi., Sch Bull, Nov 2019; 5(11): 671-680

international studies, each of which different assessment items and distinctly structured content have. This study utilized critical values for the strength of alignment among curriculum, assessment and instruction as developed by Fulmer [6].

\section{RESULTS}

Analysis and Comparison of Alignments

Myanmar Physics Alignment

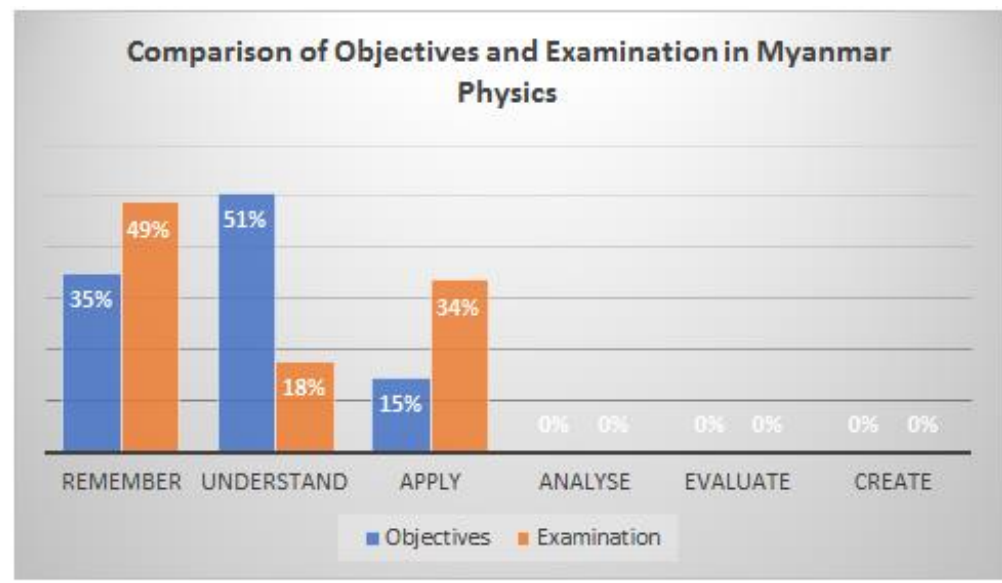

Fig-1: Comparison of objectives and examination by cognitive skills in Myanmar

Table-1: Distribution of objectives in Myanmar Physics curriculum

\begin{tabular}{|c|c|c|c|c|c|c|c|}
\hline Topic & Remember & Understand & Apply & Analyze & Evaluate & Create & Subtotal \\
\hline Mechanics & 0.096 & 0.036 & 0.024 & 0 & 0 & 0 & 0.157 \\
\hline Heat & 0.036 & 0.036 & 0.012 & 0 & 0 & 0 & 0.084 \\
\hline Waves and Sound & 0 & 0.048 & 0.012 & 0 & 0 & 0 & 0.060 \\
\hline Optics & 0.072 & 0.048 & 0.012 & 0 & 0 & 0 & 0.133 \\
\hline Electricity & 0.133 & 0.277 & 0.072 & 0 & 0 & 0 & 0.482 \\
\hline Modern Physics & 0.012 & 0.060 & 0.012 & 0 & 0 & 0 & 0.084 \\
\hline Subtotal & 0.349 & 0.506 & 0.145 & 0 & 0 & 0 & 1.000 \\
\hline
\end{tabular}

Table-2: Distribution of questions in Myanmar Physics examination

\begin{tabular}{|c|c|c|c|c|c|c|c|}
\hline Topic & Remember & Understand & Apply & Analyze & Evaluate & Create & Subtotal \\
\hline Mechanics & 0.066 & 0.013 & 0.039 & 0 & 0 & 0 & 0.118 \\
\hline Heat & 0.013 & 0.013 & 0.053 & 0 & 0 & 0 & 0.079 \\
\hline Waves and Sound & 0.013 & 0 & 0 & 0 & 0 & 0 & 0.013 \\
\hline Optics & 0.099 & 0.020 & 0.079 & 0 & 0 & 0 & 0.198 \\
\hline Electricity & 0.151 & 0.053 & 0.164 & 0 & 0 & 0 & 0.368 \\
\hline Modern Physics & 0.145 & 0.079 & 0 & 0 & 0 & 0 & 0.224 \\
\hline Subtotal & 0.487 & 0.178 & 0.335 & 0 & 0 & 0 & 1.000 \\
\hline
\end{tabular}

Figure 1 shows the overall cognitive alignment of learning objectives and their respective assessment task. Values in figure 1 are drawn from the subtotal rows in Tables 1 and 2 for objectives and examination respectively and converted to percentages. Tables 1 and 2 also provide a breakdown of proportional frequencies by topic with cognitive level. The Porter's alignment index was calculated based on the results of Tables 1 and 2, representing the distribution of levels of cognitive skills in the statements of the objectives and examination. The alignment between outcomes and examination was 0.58 , which is significantly lower than the critical value $(0.839)$ at the 0.05 level. Overall, there were no objectives or assessment tasks that required students' higher-order thinking skills (Analyse, Evaluate and Create). There was no category where the alignment between assessment task and objectives was reasonably proportioned, with the proportion in the test being higher than that in objectives in the Remember and Apply levels (35\% compared to $49 \%$ in Remember and $15 \%$ as opposed to $34 \%$ in Apply level). The one level whose proportion was higher in the objectives than that in the examination was Understand $(51 \%$ as opposed to $18 \%$ ). In terms of the quality of statements of objectives and questions, both showed emphasis on lower-order thinking skills, and thus would be in danger of encouraging rote learning and memorization as the dominant mode of student learning. 


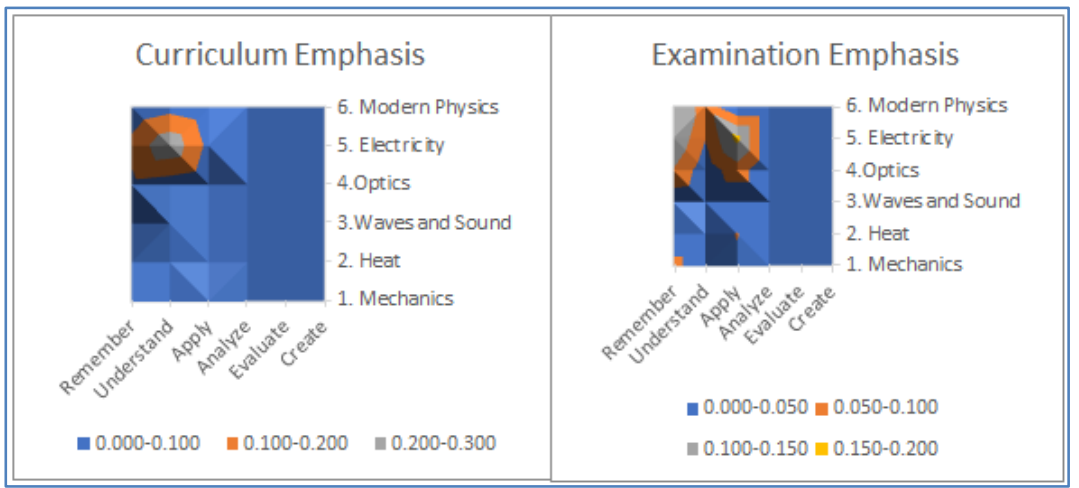

Fig-2: Comparison between curriculum emphases and examination emphases for the Myanmar Physics

Contour maps of (Figure 2) illustrate proportional frequency of cognitive levels across content areas for the objectives and examination materials in the Myanmar context. In these contour maps, blue areas show the least emphasis (ratio below 0.5 , or $5 \%$ ), orange areas indicate a moderate emphasis (ratio between 0.05 and 0.1 , or $5 \%-10 \%$ ), gray areas indicate a heavy emphasis (ratio between 0.1 and 0.15 , or $10 \%-15 \%$ ), and finally, yellow areas describe the most emphasis $(0.15-0.2$, or $15 \%-20 \%)$. Overall, the two contour maps are not similar in distribution of shading, highlighting the lack of alignment between them. In regards to the curriculum, only a moderate emphasis (gray areas) arose for Remember, Understand and Apply levels, and Electricity and Modern Physics. Whereas for the examination, the strongest emphasis (yellow area) was at the Apply level and Electricity. This result is mirrored in the Porter's alignment index of 0.58 being significantly lower than the critical value (0.839).

\section{South Australian Physics Alignment}

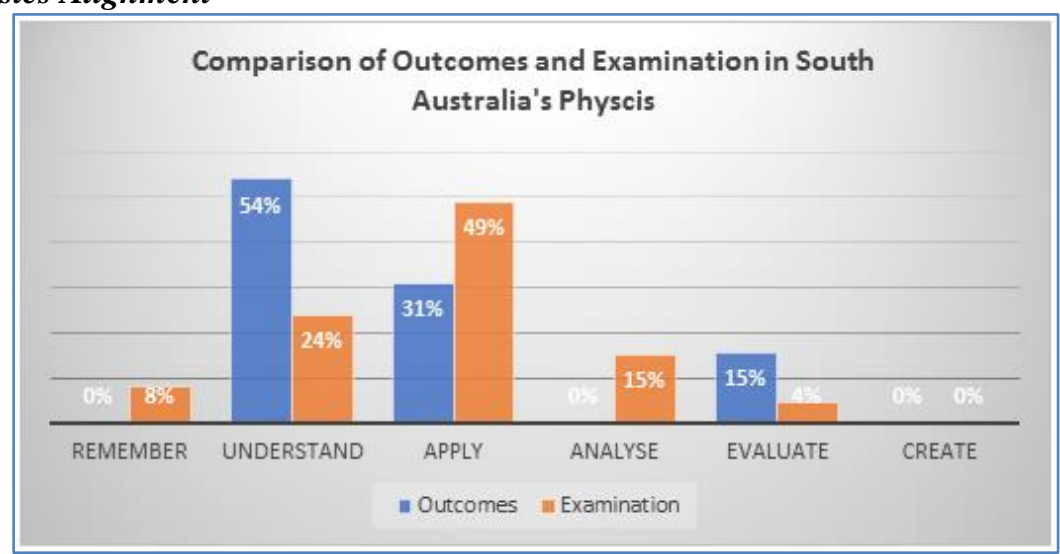

Fig-3: Comparison of outcomes and examination by cognitive skills in South Australia

Table-3: Distribution of objectives in South Australia's Physics curriculum

\begin{tabular}{|l|c|c|c|c|c|c|c|}
\hline \multicolumn{1}{|c|}{ Topic } & Remember & Understand & Apply & Analyze & Evaluate & Create & Subtotal \\
\hline Motions in Two Dimensions & 0 & 0.154 & 0.077 & 0 & 0.038 & 0 & 0.269 \\
\hline Electricity and Magnetism & 0 & 0.115 & 0.077 & 0 & 0.039 & 0 & 0.231 \\
\hline Light and Matter & 0 & 0.154 & 0.077 & 0 & 0.038 & 0 & 0.269 \\
\hline Atoms and Nuclei & 0 & 0.115 & 0.077 & 0 & 0.039 & 0 & 0.231 \\
\hline \multicolumn{1}{|c|}{ Subtotal } & 0 & 0.538 & 0.308 & 0 & 0.154 & 0 & 1.000 \\
\hline
\end{tabular}

Table-4: Distribution of questions in South Australia's Physics examination

\begin{tabular}{|l|c|c|c|c|c|c|c|}
\hline \multicolumn{1}{|c|}{ Topic } & Remember & Understand & Apply & Analyze & Evaluate & Create & Subtotal \\
\hline Motions in Two Dimensions & 0.011 & 0.033 & 0.128 & 0.039 & 0.028 & 0 & 0.239 \\
\hline Electricity and Magnetism & 0 & 0.017 & 0.089 & 0.011 & 0 & 0 & 0.117 \\
\hline Light and Matter & 0.028 & 0.011 & 0.100 & 0.033 & 0.017 & 0 & 0.189 \\
\hline Atoms and Nuclei & 0.039 & 0.178 & 0.172 & 0.067 & 0 & 0 & 0.456 \\
\hline \multicolumn{1}{|c|}{ Subtotal } & 0.078 & 0.239 & 0.489 & 0.150 & 0.044 & 0 & 1.000 \\
\hline
\end{tabular}


Figure 3 and Tables 3 and 4 show analogous findings in the South Australian context. Overall, no category exhibited strong alignment between the learning outcomes and the examination. Similar to the Myanmar Physics findings, the Porter's alignment index for the South Australian materials was 0.53, which again is significantly lower than the critical value at the 0.05 level (the critical value is 0.884 ) [6]. There were no objectives found at the Remember and Analyse levels, while $8 \%$ and $15 \%$ of examination were categorised at these levels. No objectives or examination was categorised at the Create level. More than half of the learning outcomes were categorised at Understand level and yet only 24 of questions required this level of thinking in the examination. Similarly, a higher proportion of learning outcomes at the Evaluate level were found in the outcomes compared to the examination (15\% compared to 5\%). Apply was the only level where a higher proportion was found in the examination compared to the outcomes $(49 \%$ as opposed to $31 \%$ ).

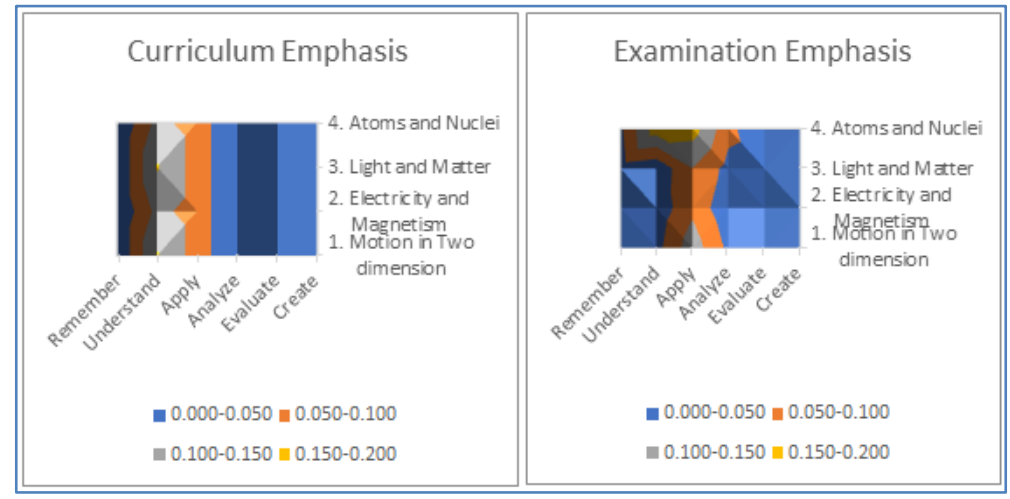

Fig-4: Comparison between curriculum emphases and examination emphases for the South Australian Physics

According to contour maps of Figure 4, the overall distributions are visually quite different, indicating a clear misalignment between them. This is once again supported by the substantially smaller value of the Porter alignment index of 0.53 than the critical value (0.884).

\section{Comparison of Physics Learning Outcomes}

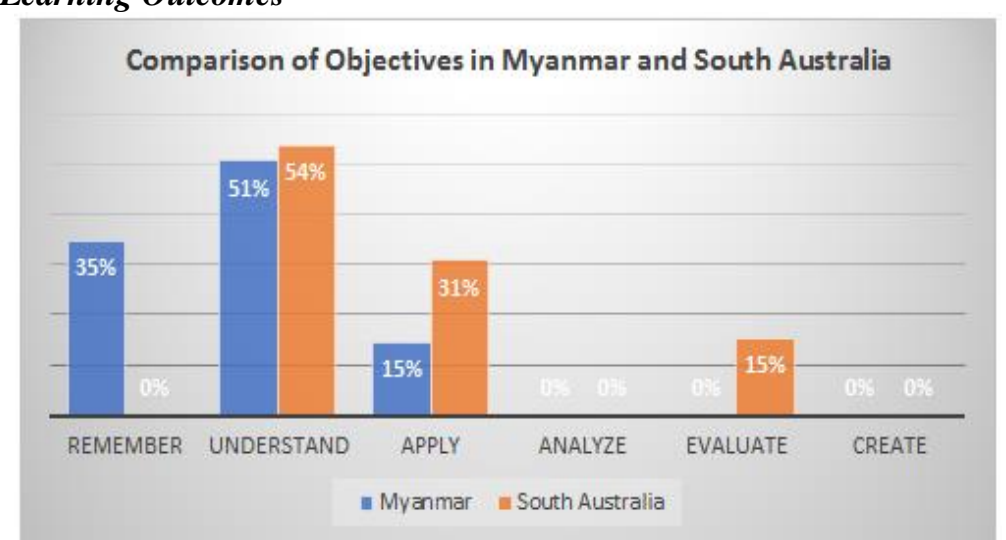

Fig-5: Comparison of emphasis on objectives between Myanmar and South Australia

Figure 5 shows the comparison of emphasis on learning objectives or learning outcomes between Myanmar and South Australia. Myanmar Physics curriculum placed a large amount of emphasis on Remember (35\%), while the South Australia curriculum had no emphasis at all. The opposite was true for the
Evaluate level, where the South Australia's objectives were categorized at this level $(15 \%)$ while no objectives were found beyond the Apply level in Myanmar. In contrast, both Myanmar and South Australia showed high emphasis for Understand (54\% compared to 51\%). 


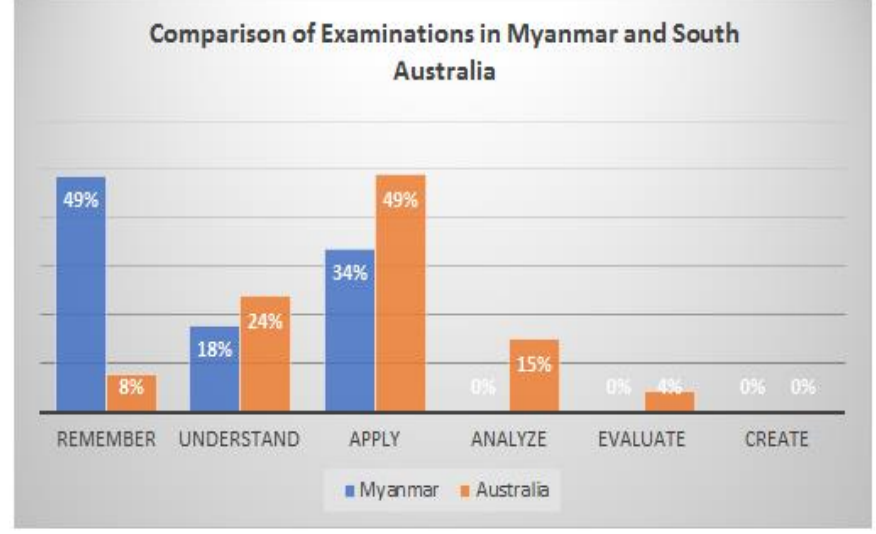

Fig-6: Comparison of emphasis on examination between Myanmar and South Australia

Figure 6 shows the comparison of emphasis on assessment tasks between Myanmar and South Australia. It was found that the Myanmar physics question set showed the strongest emphasis at the lowest cognitive level of Remember. This was in contrast to the strongest South Australia level which was Apply at 49\% (compared to the Myanmar Apply level score of $8 \%$ ). Note however the bulk of the South Australia Examination set scored in the lower cognitive levels. The South Australia's assessment task placed more emphasis on the Understand and Apply levels than did Myanmar's (24\% as opposed to $18 \%$, and $49 \%$ compared to 33\%). Even though some of South Australia's questions were found under the Analyse and Evaluate levels (15\% and 4\%), no questions were categorised beyond the Apply level in Myanmar. From these results, it is clear that the South Australia Year 12 Physics examination measured more higher-order thinking skills (Apply, Analysis and Evaluate) than the Myanmar Physics examination.

\section{DISCUSSION}

This study analysed the alignment between Myanmar and South Australia's learning objectives, learning outcomes, and examinations. In the case of the Myanmar physics curriculum, poor alignment was found between outcomes and examination (0.58 alignment is significantly lower than the critical value (0.839) indicating the materials may inadequately prepare for elements of the examination. If the cognitive range of teaching activities draws directly from the objectives (as it pedagogically should), then the delivery to students will not cognitively match with the assessment they are then exposed to. The misalignment may also disorient a student's expectations as they navigate their way towards the assessment process. The other concern arising from the Myanmar findings is the overall low cognitive level being 'hit' by both materials. Neither objectives nor assessment tasks require students' conceptual understanding of the physical concepts. Such a situation may well dissuade teachers from delivering through inquiry based learning activities, such as experiments, that are known to promote conceptual development. If this is the case, students would be fostered into a shallow learning approach where memorising formulae and equations predominates [27]. Accordingly the classroom reality behind this misalignment score becomes a point of interest to ensure that students are being provided with sufficient exposure to quality instruction, which targets higher level cognitive outcomes [28-30].

Figure 2 also shows an imbalance in the coverage across topics in the Myanmar data, for the curriculum and the examination coverage is disparately stronger for topics $4,5, \& 6$. Whether this is an intentional bias by the curriculum writers for these materials is not known, yet the authors can see no reason why such an imbalance should be present. A topic such as Mechanics is crucial for a rounded physics knowledge, so unless this is covered elsewhere (an earlier year perhaps), it is a concern to appropriate curriculum coverage.

In the South Australia physics curriculum, we again find poor alignment (the alignment index between outcomes and examination was 0.53 , which is significantly lower than the critical value $(0.884)$. This appears to be due to a greater emphasis on the higher order skills of Apply and Analyse in the examination materials in contrast to the outcome standards where Understand dominates. This mismatch raises the same concern as above. For if the cognitive level of classroom activities is drawn from the standards, the students will be under prepared for examination. Although it must be pointed out that the National standards in Australia are a recent policy addition that has been added over the top of existing state based materials. As state assessment historically emerged from state based standards, the Australian situation is complicated and ultimate conclusions difficult to draw. A further study on learning activity alignment would be needed to illuminate the situation more fully. 
The profile of cognitive level in the South Australian context, if one runs an eye across Figure 3, shows a poor profile for the outcomes as it is weighted to lower levels, but a much more balanced profile for the examination. Thus if the outcomes might be modified to align more closely with assessment then we would have both good alignment and a reasonable cognitive profile in the materials. It is again noted that Create does not feature in Figure 3; an expected result as this is a typically universal finding. It is very unusual to see assessment that targets this highest of the cognitive levels and such a situation is outside the scope of this paper. What does stand out for the South Australia context is the uniform coverage by cognitive level shown in figure 4 . The uniformity in the columns of Curriculum Emphasis is particularly strong.

When we compare the two contexts, although both show alignment concerns, it is the lower cognitive level across the Myanmar materials that stand out and to a lesser extent likewise for the South Australia objectives. Figure 5 shows clearly this situation for the objectives from both countries. Myanmar has an aggregate of $86 \%$ for the lowest two cognitive levels, whereas for South Australia the aggregate is $54 \%$. Although this makes the South Australia materials cognitively stronger, their lower level aggregate is still a majority portion (being greater than $50 \%$ ) and so both contexts are a concern. The assessment situation, Figure 6 , tells a different story. Here the Myanmar materials remain cognitively too low (aggregate of $67 \%$ for the lowest two levels), whereas the South Australia materials are more reasonably spread (lower aggregate of $32 \%$ ). Indeed, as mentioned above, the South Australia materials show a healthy cognitive distribution throughout. When looking at assessment, the curriculum writers of Myanmar may well find the South Australia materials one they might use, however the same is not to be said of their standards.

Before concluding this paper, as a final point of discussion it is worth making comparison with the findings from the alignment study of Liu et al. [13] as they also analysed the final-year of a school physics context. Similar to this study, Liu et al. found both Singapore and China showed poor alignment, whilst only New York yielded a good alignment result. It appears that poor alignment may well be more the norm across countries than not. Like South Australia, Singapore demonstrated a strong arc across cognitive levels, boding well for high cognitive learning outcomes (not surprising as they rank highly in international science attainment scores). Whereas materials from both the New York and China context over-emphasized the lower cognitive levels similarly, though not as extreme, to the Myanmar context.

\section{CONCLUSION}

Clearly there are significant discrepancies in alignment and concerns in the profiles of cognitive levels in aspects of both countries materials. The Myanmar results suggest that modifications to improve alignment between objectives and assessment are warranted and an increase in cognitive level across all topics would be beneficial. Such improvement would bring a greater coherence to assist learner orientation and raise the cognitive level of learning and so enhance higher order learning outcomes. To make inferences about student ability in conducting projects, and applying an understanding of physics concepts to reallife situations, it is suggested that Myanmar physics curriculum includes assessment that goes beyond a mere written examination. In the South Australia context alignment concerns between outcomes, a national document, and assessment, a state based document are raised. The latter demonstrated a reasonably strong arc to its cognitive level and so perhaps it is the national level documents that need to come into alignment with the existing state level documents. To this end, it would be recommended that an alignment study of assessment materials across a number of Australian states would be a useful further study that may bolster the case for modification of the National standards if the state materials show good alignment.

\section{REFERENCES}

1. Fraser, B., Tobin, K., \& McRobbie, C. J. (2011). Second international handbook of science education. Springer Science \& Business Media, 24

2. Liu, X., Zhang, B., Liang, L. L., Fulmer, G., Kim, B., \& Yuan, H. (2009). Alignment between the physics content standard and the standardized test: A comparison among the United States-New York State, Singapore, and China-Jiangsu. Science Education, 93(5), 777-797.

3. Mikelskis-Seifert, S. (n.d.). Four Decades of Research in Science Education - from Curriculum Development to Quality Improvement: From Curriculum Development to Quality Improvement. Waxmann Verlag.

4. Linn, R. L., Baker, E. L., \& Betebenner, D. W. (2002). Accountability systems: Implications of requirements of the no child left behind act of 2001. Educational Researcher, 31(6), 3-16.

5. Contino, J. (2013). A case study of the alignment between curriculum and assessment in the New York State Earth Science standards-based system. Journal of Science Education and Technology, 22(1), 62-72.

6. Fulmer, G. W. (2011). Estimating critical values for strength of alignment among curriculum, assessments, and instruction. Journal of Educational and Behavioral Statistics, 36(3), 381402. 
7. Polikoff, M. S., Porter, A. C., \& Smithson, J. (2011). How well aligned are state assessments of student achievement with state content standards? American Educational Research Journal, 48(4), 965-995.

8. Edwards, N. (2010). An analysis of the alignment of the Grade 12 Physical Sciences examination and the core curriculum in South Africa. South African Journal of Education, 30(4), 571-590.

9. Ndlovu, M., \& Mji, A. (2012). Alignment between South African mathematics assessment standards and the TIMSS assessment frameworks. Pythagoras, 33(3), 1-9.

10. Liang, L. L., Chen, X., Jiang, M., Cai, L., Du, J., \& Zhou, Y. (2017). Alignment between the National Science Curriculum standards and standarised exams at seconday school gateways. Chines Science Education in the $21^{\text {st }}$ Century: Policy, Practice, and Research, Contemporary Trends and Issues in Science Education 45, Doi 10/1007/97894-017-9864-8-10

11. Liang, L. L., \& Yuan, H. (2008). Examining the alignment of Chinese National Physics Curriculum Guidelines and 12th-grade exit examinations: A case study. International Journal of Science Education, 30(13), 1823-1835.

12. Lu, Q., \& Liu, E. (2012). Alignment between high school biology curriculum standard and the standardised tests of four provinces in China. Journal of Biological Education, 46(3), 149-164.

13. Liu, X., \& Fulmer, G. (2008). Alignment between the science curriculum and assessment in selected NY State regent's exams. Journal of Science Education and Technology, 17(4), 373-383.

14. Biggs, J., \& Tangs, C. (2007). Teaching for quality learning at University ( $3^{\text {rd }}$ edn). The society for research into Higher Education. Open University Press, England.

15. Martone, A., \& Sireci, S. G. (2009). Evaluating alignment between curriculum, assessment, and instruction. Review of Educational Research, 79(4), 1332-1361.

16. Chadwick, S. M. (2004). Current Products and Practices: Curriculum development in orthodontic specialist registrar training: can orthodontics achieve constructive alignment? Journal of Orthodontics, 31(3), 267-274.

17. Valsraj, K. M., \& Lygo-Baker, S. (2006). A balancing act: developing curricula for balanced care within community psychiatry. Advances in Psychiatric Treatment, 12(1), 69-78.

18. Shin, N., Choi, S., Stevens, S. \& Krajcik, J. (2019). The Impact of Using Coherent Curriculum on Students' Understanding of Core Ideas in Chemistry, International Journal of Science and Mathematics Education, 17, 2, pp 295-315.
19. Orlich, D. C., Harder, R. J., Callahan, R. C., Trevisan, M. S., \& Brown, A. H. (2004). Teaching strategies: A guide to effective instruction. Cengage Learning.

20. Kitchen, R. S., \& Wilson, L. D. (2004). Lessons learned from students about assessment and instruction. Teaching Children Mathematics, 10(8), 394-400.

21. South Australian Certificate of Education SACE. (2018). SACE Assessment and Reporting Guidelines. SACE Board of South Australia. Australia. Retrieved from https://www.sace.sa.edu.au/documents/652891/f2d 3a20c-e190-4a6a-a8c5-4ba954cc99a5

22. Australian Curriculum Assessment Reporting Authority (ACARA). (2017). Retrieved from

23. Anderson, L. W., Krathwohl, D. R., Airasian, P., Cruikshank, K., Mayer, R., Pintrich, P., Wittrock, M. (2001). Taxonomy for learning, teaching and assessing: A revision of Bloom's taxonomy. New York.

24. Artz, A. F., \& Armour-Thomas, E. (1992). Development of a cognitive-metacognitive framework for protocol analysis of mathematical problem solving in small groups. Cognition and instruction, 9(2), 137-175.

25. Council of Chief State School Officers. (2004). Coding procedure for curriculum content analysis. Washington, DC: Council of Chief State School Officers.

26. Porter, A. C., \& Smithson, J. L. (2001). Defining, developing, and using curriculum indicators.

Retrieved from

http://repository.upenn.edu/cpre_researchreports/69 /?utm_source=repository.upenn.edu $\% 2 \mathrm{Fcpre}$ _resea rchreports\%2F69\&utm_medium=PDF\&utm_camp aign=PDFCoverPages

27. Krathwohl, D. R. (2002). A revision of Bloom's taxonomy: An overview. Theory into Practice, 41(4), 212-218.

28. Huffman, D. (1997). Effect of explicit problem solving instruction on high school students' problem-solving performance and conceptual understanding of physics. Journal of Research in Science Teaching, 34(6), 551-570.

29. Stevens, F. I. (1996). Opportunity to learn science: Connecting research knowledge to classroom practices. Laboratory for Student Success, the MidAtlantic Regional Educational Laboratory at Temple University, Center for Research in Human Development and Education.

30. Wang, J. (1998). Opportunity to learn: The impacts and policy implications. Educational Evaluation and Policy Analysis, 20(3), 137-156.

31. Porter, A. C. (2002). Measuring the content of instruction: Uses in research and practice. Educational Researcher, 31(7), 3-14. 